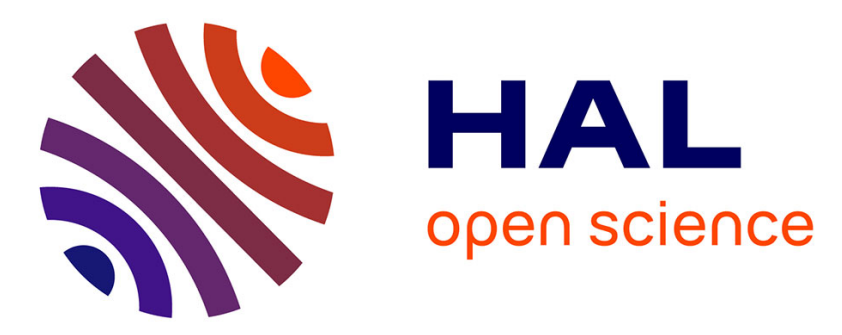

\title{
Evolution récente des paramètres cryo-climatiques et des teneurs isotopiques des précipitations dans les Andes boliviennes: La Paz et Glacier du Zongo
}

Robert Gallaire, J.D. Taupin, Anne Coudrain

\section{- To cite this version: \\ Robert Gallaire, J.D. Taupin, Anne Coudrain. Evolution récente des paramètres cryo-climatiques et des teneurs isotopiques des précipitations dans les Andes boliviennes: La Paz et Glacier du Zongo. Hydrological Sciences Journal, 2010, 10.1080/02626661003747366 ird-01223158}

\section{HAL Id: ird-01223158 \\ https://hal.ird.fr/ird-01223158}

Submitted on 2 Nov 2015

HAL is a multi-disciplinary open access archive for the deposit and dissemination of scientific research documents, whether they are published or not. The documents may come from teaching and research institutions in France or abroad, or from public or private research centers.
L'archive ouverte pluridisciplinaire $\mathbf{H A L}$, est destinée au dépôt et à la diffusion de documents scientifiques de niveau recherche, publiés ou non, émanant des établissements d'enseignement et de recherche français ou étrangers, des laboratoires publics ou privés. 


\section{Evolution récente des paramètres cryo-climatiques et des teneurs isotopiques des précipitations dans les Andes boliviennes: La Paz et Glacier du Zongo}

RoBert GALLAIRE ${ }^{1}$, JEAN-DENIS TAUPIN ${ }^{2}$, ANNE COUDRAIN ${ }^{2}$

Avec la participation de CARLOS DIAS ${ }^{3}$

1IRD, Laboratoire HydroSciences Montpellier (UMR 5569, UM II), IRD, Casilla 18-1209, Lima 18, Pérou robert.gallaire@ird.fr

${ }^{2}$ IRD, Laboratoire HydroSciences Montpellier(UMR 5569, UM II), MSE, 300 avenue Jeanbrau, 34000, Montpellier, France

${ }^{3}$ SENAMHI, calle Reyes Ortiz, n41, La Paz, Bolivia

Résumé : A proximité de La Paz, les indicateurs marquants de la tendance climatique sont a) une augmentation de la température de ré-analyse à $500 \mathrm{hPa}$ de $0,017^{\circ} \mathrm{C} . \mathrm{an}^{-1}$ entre 1973 et 2004, b) à la station météorologique Laïca Cota, entre 1995 et 2004, une élévation de la température de $0,03^{\circ} \mathrm{C} . \mathrm{an}^{-1}$, une diminution de $1^{\prime}$ humidité relative de $0,6 \%$.an $\left.{ }^{-1}, \mathrm{c}\right)$ une augmentation du $\delta^{18} \mathrm{O}$ des précipitations de $0,26 \%$.an-1 et de leur excès en deutérium de $0,1 \%$.an-1. Une des principales conséquences de cette tendance est un recul du front du glacier Zongo de 12 m.an ${ }^{-1}$ entre 1991 et 2004 (réduction de 2\% par an des surfaces glaciaires de 1997 à $2004: 1,84 \mathrm{~km}^{2}$ en 2004).

L'analyse temporelle concomitante des données montre que la variabilité des conditions ENSO a des conséquences en phase à l'échelle infra-annuelle pour la température, l'humidité relative, la précipitation, le bilan glaciaire, et des conséquences en déphasage, sur des périodes dépassant l'année, pour le rapport isotopique des précipitations andines.

Mots clefs: Andes ; Bilan et débit glaciaire; Précipitation ; Humidité relative ; Isotopes stables ; Indice d'Oscillation Sud (S.O.I.) ; Températures au sol et de ré-analyse. 


\section{INTRODUCTION}

Les derniers résultats du GIEC 2007 (Groupe Intergouvernemental d'experts sur l'Evolution du Climat, ou IPCC) sur la caractérisation du changement climatique global illustrent le besoin d'accroître cette information dans l'hémisphère sud. L'étude des variables climatiques et glaciologiques depuis 1991 - ou reconstituées depuis 1973 - qui est présentée dans ce travail sur les Andes boliviennes est donc une contribution à l'amélioration générale de la connaissance dans ce domaine.



Fig. 1 : Localisation de la zone d'étude et principales caractéristiques climatiques et altitudinales.

La climatologie de l'altiplano andin bolivien, situé en région intertropicale (figure 1), et particulièrement celle de la zone d'étude (couloir d'environ $200 \mathrm{~km}^{2}$ du glacier Zongo à la station météorologique de Laïca Cota - La Paz), est conditionnée par l'entrée des masses d'air humide d'origine atlantique. Ces masses d'air, après avoir circulé au-dessus de l'Amazonie, portent 
l'héritage d'une variabilité climatique, tant à l'échelle annuelle (saison sèche et saison des pluies très contrastée), qu'interannuelle avec le phénomène ENSO (El Niño Southern Oscillation): Phénomène climatique, et océanique, lié aux variations australes de la pression atmosphérique et de la température océanique du Pacifique sud, mesurées entre le nord de l'Australie (Darwin) et les côtes tropicales de l'Amérique du sud (Equateur-Pérou). En situation El Niño, le Pacifique est plus chaud $\left(2\right.$ à $\left.4^{\circ} \mathrm{C}\right)$ et dépressionnaire, en raison du basculement de la thermocline (contraste thermique des eaux chaudes, de surface, et froide de profondeur, de l'océan) vers le continent américain. Cette situation provoque sur les côtes équatoriennes et nord péruviennes des précipitations importantes à catastrophiques (Niño 97-98). Au même moment il semble que les entrées d'air d'origine atlantique aient plus de difficultés pour atteindre les Andes et les hautes surfaces intra-andines, entre les Cordillères Orientale et Occidentale (Altiplano), où les précipitations diminuent et où les températures s'élèvent. En situation La Niña le phénomène inverse s'observe, le basculement de la thermocline vers l'Australie maintient les eaux chaudes du Pacifique à l'ouest, les remontées d'eau froide (Upwelling) sont facilitées à l'est, où elles renforcent l'aridité des côtes sud américaines. A l'intérieur, sur l'Altiplano, les entrées d'air atlantique apparaissent facilitées durant la saison des pluies, les précipitations sont plus abondantes et les températures plus fraîches.

Plusieurs études sur cette région d'altitude andine ont montré son potentiel à caractériser des variabilités climatiques régionales et en particulier l'évolution des conditions climatiques le long du trajet des masses d'air sur le bassin amazonien (Hoffman et al., 2003 ; Vimeux et al., 2005), depuis leur origine atlantique, jusqu'aux lieux de précipitation et de stockage partiel dans les Andes. L'objet de ce travail est a) d'étudier l'évolution récente de différentes variables climatiques et du bilan d'un glacier proche, b) d'analyser leur synchronisme ou décalage temporel afin de caractériser leurs connexions dans un contexte ENSO variable, c) d'évaluer le potentiel des isotopes stables des précipitations, comme indicateur indirect de cette variabilité ENSO du climat. 


\section{DONNEES UTILISEES}

Les données utilisées sont des mesures au sol (variables climatiques de température et d'humidité, bilans de masse et débits du bassin du Zongo, englacé aux deux tiers), des indices ENSO et des températures de ré-analyse. Les données utilisées couvrent deux périodes en fonction des disponibilités: celle de 1973 à 2004, en ce qui concerne les débits et les températures de ré-analyse, celle de 1995 à 2004 pour les mesures isotopiques et climatiques concomitantes. Les données de modèles et d'indices ENSO ont été, quant à elles, collectées sur le web.

Laïca Cota, station météorologique du Service national météo-hydrologique bolivien (Senamhi), située au centre de $\operatorname{La} \operatorname{Paz}\left(16^{\circ} 29^{\prime} 56^{\prime}\right.$ 'S, 6807’23'’O, $\left.3635 \mathrm{~m}\right)$, capitale de la Bolivie, fait partie, depuis septembre 1995, du Global Network for Isotopes in Precipitation (G.N.I.P. IAEA/WMO 2004- http://nds121.iaea.org/wiser/), à l'initiative commune de l'Institut de Recherche pour le Développement (IRD) et du Senamhi qui en assurent le suivi. Les pluies sont recueillies à la fin de chaque événement pluvieux et stockées dans un bidon étanche, à l'abri de la chaleur. Chaque fin de mois, est extrait du bidon collecteur un échantillon qui représente une pondération des différents événements pluvieux survenus au cours du mois (une homogénéisation air-eau par vigoureuse agitation permet de pallier, en grande partie, les phénomènes d'évaporation développés au sein du bidon durant le stockage mensuel). Cet échantillon, conditionné hermétiquement dans une bouteille fumée à double bouchage, est envoyé à l'A.I.E.A. (Agence Internationale de l'Energie Atomique) de Vienne, pour analyse des isotopes stables $\left({ }^{18} \mathrm{O}, \mathrm{D}\right)$. Les résultats isotopiques sont donnés avec une précision de $0,05 \%$ et $0,6 \%$ (respectivement pour l'oxygène 18 et le deutérium). Parallèlement à l'échantillonnage de la pluie mensuelle, les paramètres climatiques mesurés à la station Laïca Cota (quantité de précipitation, température et humidité relative) ont été relevés pour la même période de septembre 1995 à décembre 2004.

A la même échelle de temps et de durée, les données de bilan hydrologique et de bilan de masse du glacier Zongo, descendant du Huayna Potosi, situé à quarante kilomètres au nord-est de La Paz $\left(16^{\circ} 15^{\prime} \mathrm{S}-68^{\circ} 10^{\prime} \mathrm{O}\right)$, ont été recueillies afin de les comparer aux données isotopiques de la station 
Laïca Cota. Ce glacier suivi depuis 1991, dans le cadre de collaborations franco-boliviennes, présentait en 2004 une surface englacée de $1,84 \mathrm{~km}^{2}$ (61,3\% du bassin), comprise entre 4900 et $6088 \mathrm{~m}$ d'altitude. Les débits à la sortie du bassin glaciaire du Zongo, reconstitués depuis 1973 (Pouyaud et al., 1999), puis mesurés à partir de 1991, ont été comparés sur la période 1973-2004 aux températures de ré-analyse NCEP-NCAR (Kalnay et al., 1996). Ces températures sont issues de modèles climatiques globaux dont les données d'entrée proviennent de nombreuses sources différentes: stations météorologiques terrestres et maritimes embarquées, radiosondages atmosphériques à partir de ballons sonde, d'avions, de satellites. Ces données de ré-analyse couvrent la planète par maille carrée de $2,5^{\circ}$ et pour 17 nivaux de pression entre 10 et $1000 \mathrm{hPa}$. Les données utilisées dans notre étude sont celles du niveau $500 \mathrm{hPa}$ (correspondant à l'altitude $5600 \mathrm{~m}$ ), de la maille carrée couvrant le glacier du Zongo, dont les coordonnées sont : $15^{\circ}-17.5^{\circ} \mathrm{S}$ et $70^{\circ}-67.5^{\circ} \mathrm{O}$ (http://www.cdc.noaa.gov/cdc/data.ncep.reanalysis.tropopause.html).

Enfin l'ensemble de ces données issues de la mesure et de la modélisation ont été comparées, sur la période 1995-2004, au signal ENSO, pour lequel l'Indice d'Oscillation Australe (http://www.cru.uea.ac.uk/ftpdata/soi.dat) a été retenu, afin d'identifier, particulièrement durant les évènements extrêmes, une évolution simultanée possible du comportement isotopique des précipitations et de la réaction physique du glacier.

Dans le domaine isotopique l'excès en deutérium a été utilisé pour essayer de comprendre la transformation de la masse d'air à l'origine de la précipitation recueillie à La Paz. Cet excès en deutérium est défini par la relation liant à l'équilibre l'oxygène 18 et le Deutérium dans la molécule d'eau $\left(\mathrm{d}=\delta \mathrm{D}-8 \delta{ }^{18} \mathrm{O}\right)$, il signe 1) les conditions atmosphériques de la région océanique où s'est produite l'évaporation à l'origine de la précipitation mesurée (Dansgaard, 1964), et également 2) dans les processus hors équilibre (évaporation océanique, formation des cristaux de glace...) l'effet cinétique qui, à l'évaporation, appauvrit moins la vapeur formée en deutérium qu'en oxygène 18 . Ce fractionnement cinétique dépend des conditions d'évaporation : température, mais aussi humidité relative, liée en particulier à la vitesse du vent. Les modèles de distillation et de circulation 
générale de l'atmosphère, incluant les isotopes stables, montrent que le signal de l'évaporation, dans l'excès en deutérium, est conservé le long de la trajectoire des masses d'air. Si ce signal évolue il enregistre donc un changement des conditions d'évaporation le long de la trajectoire.

\section{MODES DE CALCUL ET D'EXPRESSION}

Dans cette étude, le pas de temps considéré est celui du mois qui correspond à celui de l'analyse isotopique des pluies de la station de Laïca Cota et permet de caractériser les variations infraannuelles et inter-saisonnières. Le bilan de masse de la zone d'ablation du glacier Zongo est exprimé en gain ou perte, équivalent hauteur d'eau en mètre. Il est calculé, à partir des mesures mensuelles d'émergence du réseau de balises qui contrôle l'évolution temporelle de la surface englacée (mesures d'émergences associées à des mesures annuelles de densité) dans les zones d'ablation et d'accumulation (Francou et Pouyaud., 2004).

Enfin, à l'analyse des données brutes mensuelles ont été ajoutées des droites de tendance, et pour certaines variables, un lissage par moyenne mobile (sur 13 mois), afin de mieux faire ressortir leur évolution à moyen terme.

\section{EXAMEN DES DONNEES, SYNCHRONISMES et DECALAGES TEMPORELS}

\section{Evidence de l'évolution des conditions climatiques dans les Andes boliviennes}

Une des conséquences les plus sensibles du changement climatique actuel est l'augmentation des températures à l'échelle globale d'environ $0,74^{\circ} \mathrm{C}$ sur un siècle (1905-2006) d'après le rapport du GIEC 2007, soit $0,074^{\circ} \mathrm{C}$ par décennie, avec une accélération pour la dernière en date, puisque sur la période 1995-2006 l'augmentation décennale est presque deux fois plus importante $\left(0,13^{\circ} \mathrm{C}\right)$. Cette tendance récente est confirmée par le fait que onze des douze dernières années figurent au palmarès des années les plus chaudes depuis 1850, début de l'enregistrement des températures de surface (GIEC, 2007). Qu'en est-il dans la zone d'étude? A l'observatoire géophysique San Calixto, enclavé dans le centre historique de La Paz (16²9'43"S-687'57"O-3658 m), et proche de Laïca Cota, les températures moyennes annuelles marquent une augmentation plus sensible encore de 
$2,1^{\circ} \mathrm{C}$ entre 1937 et 1997 , qui représente une élévation décennale de $0,35^{\circ} \mathrm{C}$ sans aucun doute liée partiellement à l'effet de cité, mais qui atteste d'un phénomène andin non moins sensible que sur le reste de la planète (Segaline Nieto et Cabre, 1988).

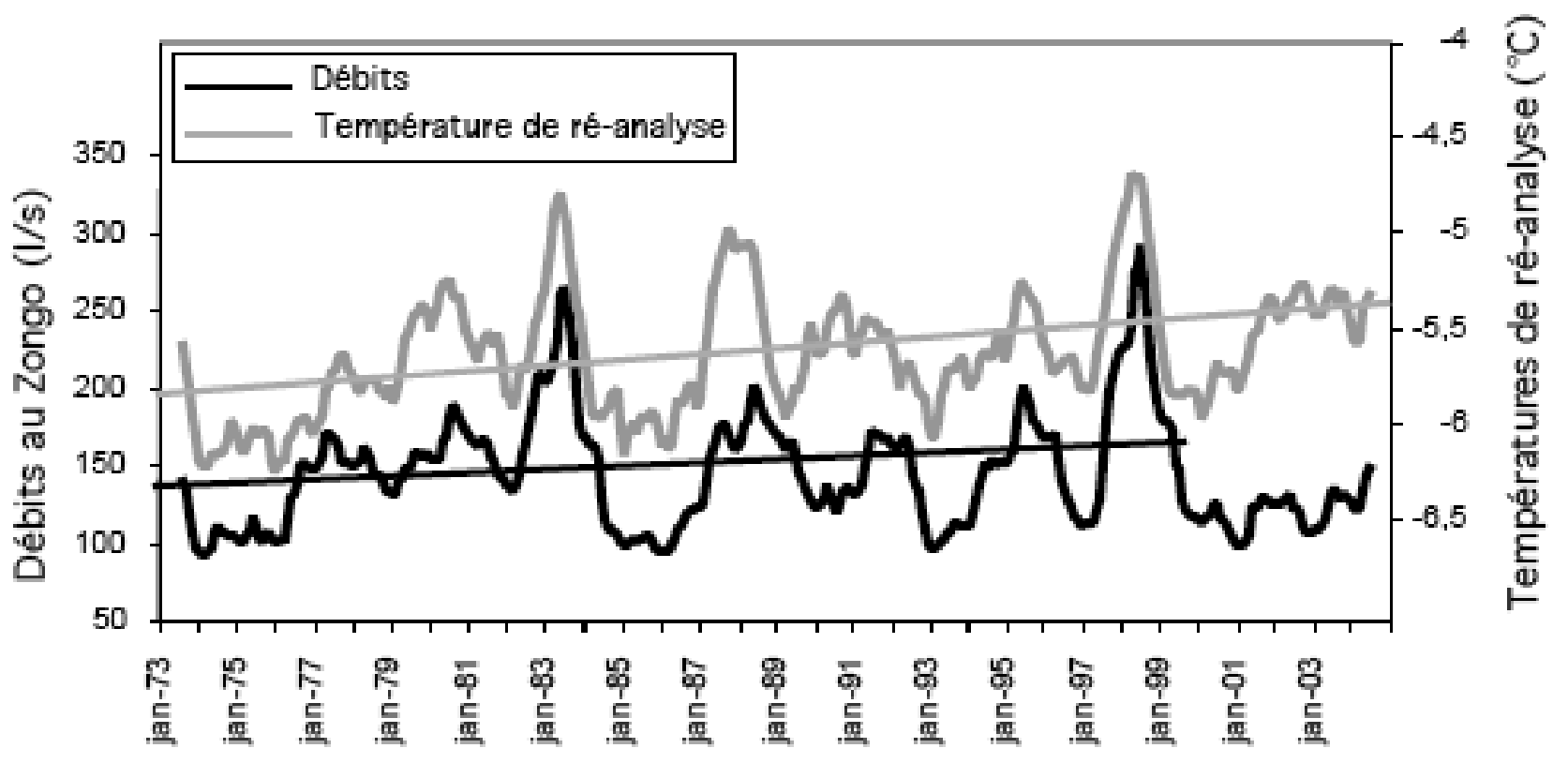

Fig. 2 Evolution des températures mensuelles de ré-analyse à $500 \mathrm{hPa}$ et des débits du glacier Zongo durant la période 1973-2004 (moyennes mobiles sur 13 mois) et droite de tendance associée.

Les températures relevées durant la période récente 1996-2004 à la station météorologique Senamhi du centre ville (Laïca Cota), située sur le site aéré d'un éperon de badlands fluvio-glaciaires dominant la vieille ville d'une centaine de mètres, montrent une augmentation de $0,3^{\circ} \mathrm{C}$, soit une tendance décennale de $0,33^{\circ} \mathrm{C}$ (figure 3), logiquement proche de celle de San Calixto. Les températures de ré-analyse NCEP, au dessus du glacier Zongo (à 5600 m d'altitude) confirment une tendance au réchauffement d'environ $0,5^{\circ} \mathrm{C}$ sur les 30 dernières années (figure 2), soit une élévation décennale moyenne de près de $0,17^{\circ} \mathrm{C}$. Ces augmentations de température à La Paz sont environ deux fois supérieures à celles des températures mesurées et de ré-analyse à l'échelle mondiale. Même si la croissance de la ville de La Paz peut avoir contribué au niveau de températures de San Calixto et de Laïca Cota, leurs valeurs $\left(+0,35 ;+0,33^{\circ} \mathrm{C}\right.$ par décennie $)$ sont conformes à celles présentées par Vuille et al. (2000) pour la région des Andes centrales à partir de mesures de 270 stations terrestres et de températures de ré-analyse sur la tranche 650-600 hPa $(0,32-$ 
0,34 ${ }^{\circ} \mathrm{C} /$ décennie) pour la période $74-98$. Bien que les hausses de température semblent un peu plus fortes dans les basses couches, dues à l'interaction probable sol-atmosphère, ces résultats prouvent bien l'existence d'un réchauffement des différentes couches atmosphériques dans la région des Andes Centrales.

Un autre paramètre corrobore une évolution climatique : L'humidité relative. Au cours de la période 1995-2005, elle montre à la station de Laïca Cota une diminution de 5,7\% (figure 3), alors que, sur la même période, la précipitation ne présente pas de tendance significative à la baisse (figure 3 ). Dans certaines régions d'Amérique du Sud une évolution de la précipitation a bien été constatée : augmentation dans la zone nord ouest amazonienne de la Colombie, diminution dans la zone centrale péruvienne et sud sud-ouest bolivienne (Espinoza et al., 2009). Une des conséquences les plus manifestes de l'évolution du climat dans les Andes est le retrait des glaciers qui s'accompagne d'une augmentation du débit de leurs bassins (Sicart et al ., 2003 ; Pouyaud et al., 2005 ; Francou et Vincent, 2007). Dans la zone d'étude, durant la période 1991-2004, le recul du front du glacier Zongo est en moyenne de $12 \mathrm{~m} . \mathrm{an}^{-1}$, l'épaisseur de sa zone d'ablation diminue en moyenne de 0,8 m.an-1, et la réduction de la surface glaciaire de 2\% par an (période 1997-2004). Cette diminution de volume des glaces s'accompagne d'une augmentation des débits à la station hydrologique « 4830 » qui contrôle les écoulements au front du glacier (figure 2). L'ablation glaciaire s'effectue en grande partie sous forme de fusion, $80 \%$ en moyenne, les $20 \%$ restants étant prélevés par sublimation. Les écarts sont néanmoins importants pour la sublimation d'une année à l'autre: 34\% de l'ablation au cours de La Niña 96-97, pour seulement 2,9\% au cours du El Niño 97-98 qui a suivi, (Wagnon et al, 2001). Le bilan d'énergie à la surface du glacier est capital, et l'humidité favorise le phénomène de sublimation qui nécessite beaucoup plus d'énergie que celui de la fusion. Les débits du glacier apparaissent remarquablement corrélés, jusqu'en 2001, avec les températures de ré-analyse à $500 \mathrm{hPa}$ du Zongo. Ces résultats sont conformes à ceux obtenus en Cordillère Blanche au Pérou (Pouyaud et al., 2005). Cependant les moyennes mobiles sur 13 mois des valeurs mensuelles de températures de ré-analyse et des débits du glacier Zongo ne présentent, sur la 
période 1973-2004, qu'un coefficient de détermination $\mathrm{R}^{2}$ de 0,53 , alors qu'il était de 0,67 sur la période 1973-2001, avant la constance des débits enregistrés en 2002-2003 malgré l'augmentation de la température. La cause principale de ce décrochage peut être imputée à La Niña qui, à partir de l'année 2000, a provoqué au Zongo d'importantes chutes de neige, tant sur le glacier que sur les moraines avoisinantes, figeant durant au moins deux ans, sous forme solide, une grande quantité de la précipitation. Le glacier durant cette période a bénéficié d'un albedo élevé qui l'a protégé de la fusion, et cela malgré la phase El Niño (d'intensité faible, mais prolongée) qui a suivi. L'eau stockée sous forme solide, sur et autour du glacier (moraines), n'a été que très progressivement restituée aux écoulements.

Les figures 2 et 3 montrent que les forts ENSO de 1982-83 et 1997-98 ont entraîné une notable augmentation des températures (mesurée et modélisée). Cette augmentation de température, associée à une précipitation plus faible (moyenne de la période 1937-1996 à San Calixto: 563 mm, précipitations de 1982 et 1983 : respectivement $479 \mathrm{~mm}$ et $405 \mathrm{~mm}$ ), ont eu pour conséquence un affaiblissement de la valeur de l'albedo. L'absorption radiative plus élevée sur le glacier, suite à cet affaiblissement de l'albedo, a accéléré la fusion de la glace, d'où une augmentation des débits à l'exutoire (Ribstein et al., 1996 ; Sicart et al., 2007). Phénomène récurrent dans les Andes Centrales depuis plus de deux décennies : les bassins versants glaciaires produisent plus d'eau qu'ils n'en reçoivent.

\section{Relation entre variabilité isotopique et variabilité climatique}

En zone tropicale, la relation directe entre composition isotopique des précipitations et paramètres climatologiques est faible (Rosanski et Araguas-Araguas, 1995). En zone de moyenne et haute latitude, le schéma classique est celui d'une diminution de la teneur en isotopes lourds pendant la saison hivernale en raison de températures fraîches, de précipitations notables et d'humidité relative proche de la saturation, (phénomène inverse en été). Ce scénario est contrarié en milieu intertropical par le fait que la saison des pluies coïncide avec la saison estivale où les températures sont les plus élevées de l'année. 


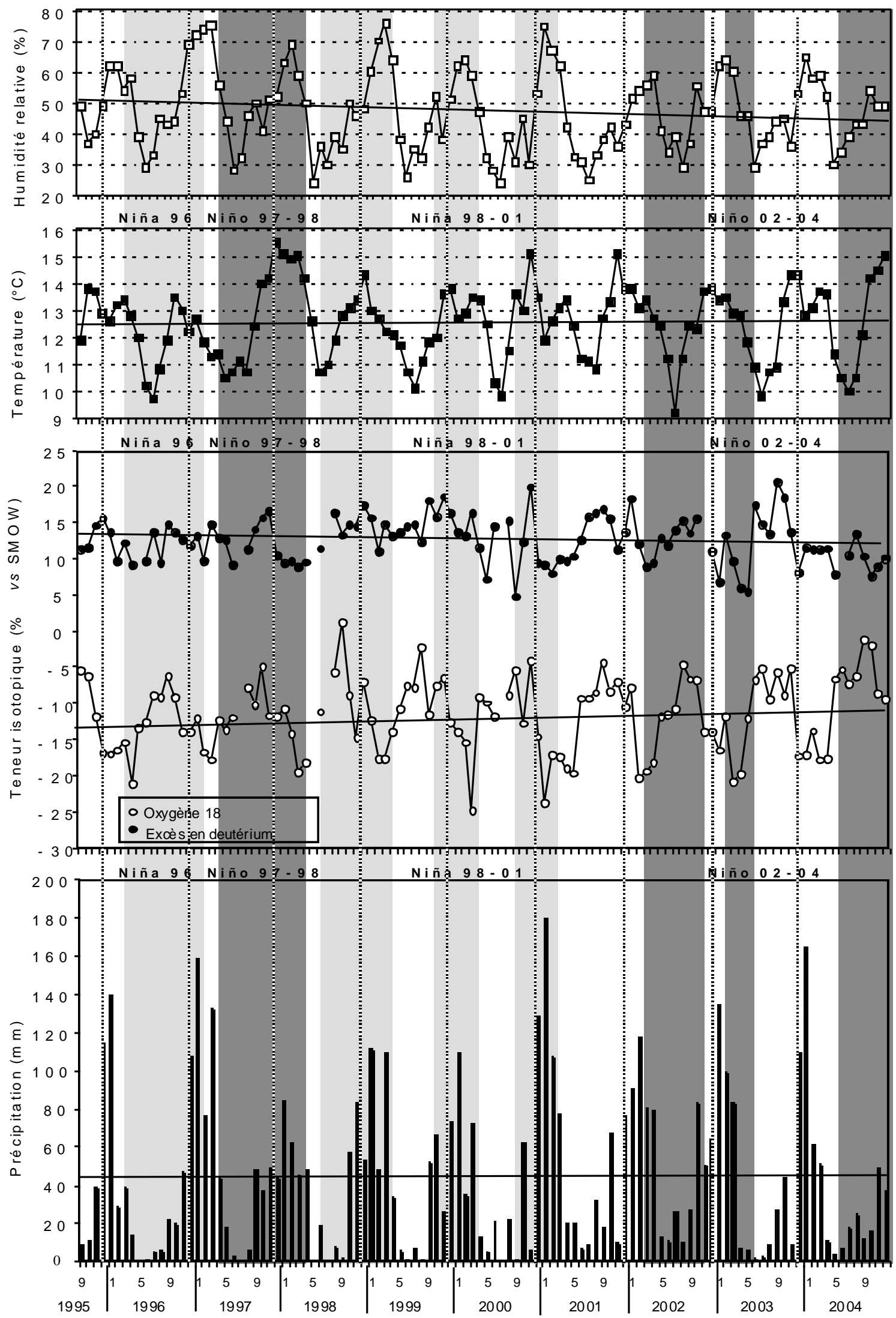

Fig. 3 Données mensuelles, à la station de Laïca Cota entre 1995 et 2004, des teneurs en Humidité relative, Température, Oxygène 18, Excès en deutérium, et hauteur des pluies. Durant la période. d'étude, les phases modérées à fortes La Niña et El Niño (valeurs S.O.I.) sont symbolisées en gris clair et gris foncé, respectivement. Pour les intensités de ces phases se reporter à la figure 7. 
D'autre part, la faible amplitude annuelle des températures $\left(<6^{\circ} \mathrm{C}\right.$ à $\left.\mathrm{La} \mathrm{Paz}\right)$ ne permet pas d'expliquer la variabilité de la teneur isotopique (25\%) relevée à Laïca Cota (Vimeux et al, 2005).

La figure 4 montre par ailleurs que cette variabilité des teneurs mensuelles en oxygène 18, obtenues au cours des dix années de mesures, est mieux expliquée par la quantité précipitée et l'humidité relative, que par les températures


Fig. 4 Valeurs mensuelles de paramètres climatiques en fonction de l'Oxygène 18 des précipitations: a) Quantité de pluie vs Oxygène 18 ; b) Oxygène 18 vs Température au sol; c) Oxygène 18 vs Humidité relative (station Laïca Cota).

L'effet isotopique de masse des précipitations est un phénomène physique qui résulte de la vidange progressive de la masse nuageuse. Il se traduit par un appauvrissement en isotopes lourds des précipitations directement lié au degré de vidange de la masse d'air, puisque ce sont les atomes lourds (oxygène 18, deutérium) qui quittent préférentiellement la masse d'air à la condensation. Mais l'humidité relative contrôle aussi, sous le nuage, lors de la précipitation, la composition 
isotopique des gouttes d'eau traversant cet espace: plus l'atmosphère est asséchée sous le nuage, plus les gouttes d'eau s'évaporent, provoquant un enrichissement en isotopes lourds de leur contenu, puisqu'à l'évaporation se sont les atomes légers (oxygène 16 et hydrogène) qui quittent en premier le condensat. Ainsi les deux phénomènes fonctionnent plus ou moins de façon antagoniste sur la composition isotopique des précipitations.

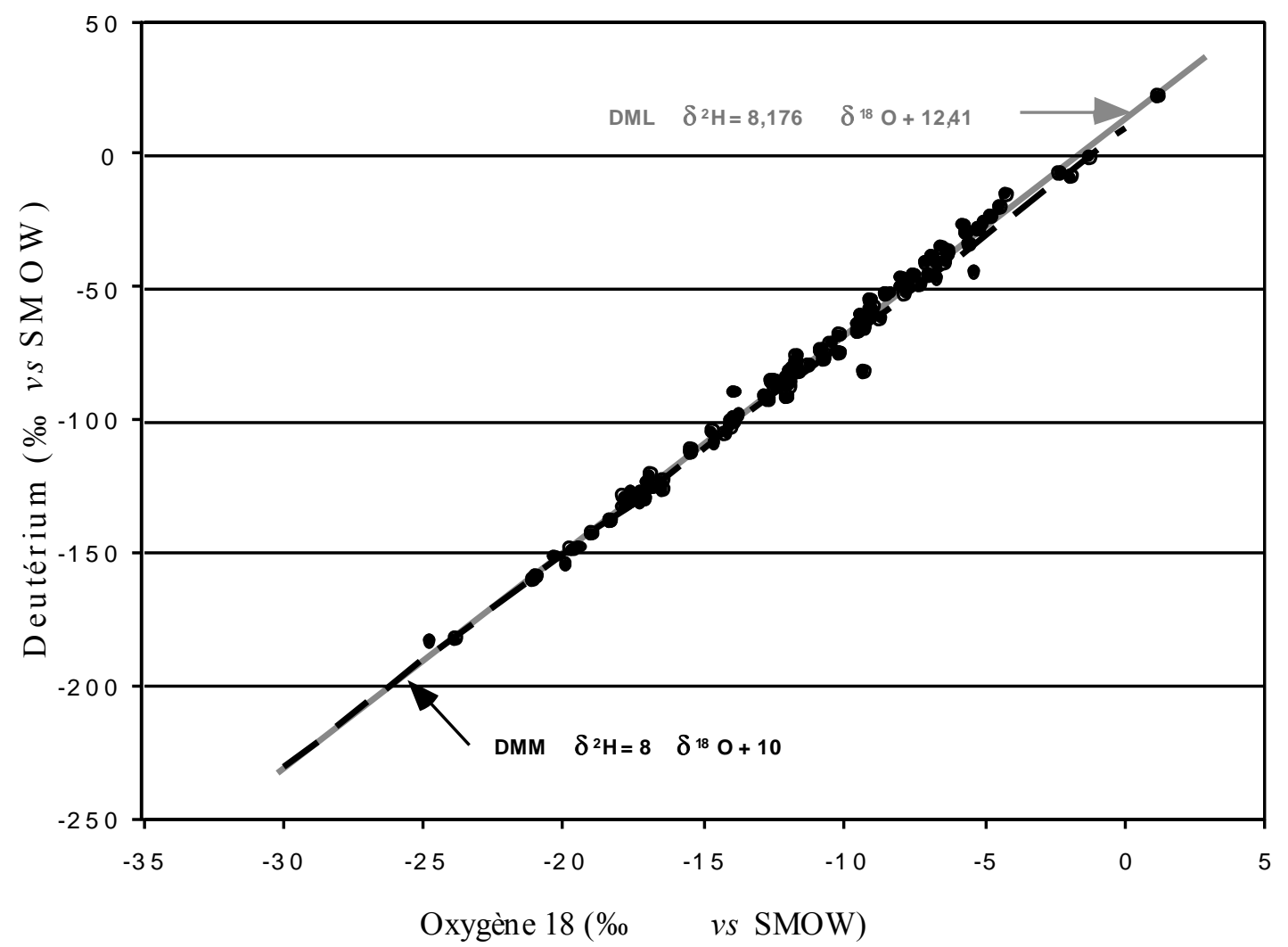

Fig. 5 Relation Deutérium vs Oxygène 18 à Laica Cota. Représentation de la DMM et de la DML. Les points au dessus de la DMM ont un excès en deutérium > 10, signe de conditions de recharge en vapeur de l'atmosphère lié à du recyclage continental, principalement.

Sur la figure 5 (deutérium vs oxygène 18), les 105 valeurs mensuelles des pluies de Laïca Cota définissent une Droite des eaux Météoriques Locale (DML) de pente $(8,18)$, légèrement supérieure à la pente de la théorique Droite Météorique Mondiale $\left(\mathrm{DMM} ; \delta \mathrm{D}=8 \delta^{18} \mathrm{O}+10\right)$ et d'ordonnée à l'origine 12,4\%o. La déviation entre ces deux droites indique des conditions atmosphériques différentes de la région source des précipitations et/ou du site de prélèvement (Craig, 1961, Merlivat et Jouzel, 1979). Dans notre cas, les précipitations sont issues de masses d'air tropical 
circulant de l'Océan Atlantique à travers le bassin amazonien (Aceituno et Montecinos, 1993 ; Vuille et al., 2000; Garreaud et al., 2003; Ronchail et Gallaire, 2006).

Deux mécanismes de même type (la re-évaporation), mais d'échelles différentes, ainsi qu'un forçage altitudinal (Gonfiantini et al., 2001), peuvent expliquer la valeur d'excès en deutérium supérieure à 10 (valeur de la source océanique) des précipitations mensuelles :

Un mécanisme régional correspondant au transfert des masses d'air au-dessus du bassin amazonien : le mélange avec de la vapeur d'eau provenant de re-évaporation au-dessus des zones d'inondation amazonienne ou de la vapeur issue de la reprise partielle par évaporation des gouttelettes interceptées par la canopée est vraisemblablement le principal mécanisme d'augmentation de l'excès en deutérium, car la transpiration des plantes n'est pas fractionnante dans les conditions d'équilibre (Salati et al., 1979 ; Gat et Matsui, 1991, Taupin et al., 2000).

Un mécanisme local lié aux conditions de précipitation en atmosphère particulièrement soussaturée d'altitude: Le phénomène de virga, associé à celui d'une évaporation partielle des gouttelettes d'eau. "En météorologie (WMO, 1956) le phénomène de «virga» désigne toute forme de précipitation, n'atteignant pas le sol. Selon la température, il est composé de cristaux de glace ou de gouttes liquides qui subliment ou s'évaporent sous un nuage en passant au travers d'une atmosphère sous-saturée. Ce phénomène est fréquent dans les régions arides en raison d'un déséquilibre important de pression partielle entre l'élément précipité et l'atmosphère de l'événement."

L'évaporation totale des gouttes d'eau (phénomène de virga) enrichit la vapeur d'eau formée en isotope lourd par rapport à la vapeur originelle ayant donné naissance à la précipitation, mais n'affecte pas l'excès en deutérium. En revanche si l'évaporation est partielle on se retrouve dans le cas d'un fractionnement cinétique conduisant à élever la valeur de l'excès en deutérium de la vapeur ambiante (Taupin et al., 2000).

Enfin les contreforts andins, en obligeant l'air amazonien à s'élever de près de 5000 mètres, participent certainement à l'augmentation de l'excès en deutérium des précipitations altiplaniques (Gonfiantini et al., 2001). 
Dans la zone d'étude, en dehors du cœur de la saison des pluies, les humidités relatives peuvent atteindre des valeurs extrêmement faibles, inférieures à 10\%, qui montrent la potentialité de reprise évaporatoire très forte des gouttes d'eau. Les quelques événements pluvieux qui ont lieu au cours de la saison sèche, ou au début de la saison des pluies, au moment où les humidités relatives sont les plus faibles de l'année, produisent en effet les valeurs d'excès en deutérium les plus élevées (figure 7).

Les valeurs d'excès en deutérium supérieures à 10 des précipitations de la capitale bolivienne, héritage d'influences régionales amazoniennes, sont donc susceptibles d'être renforcées par des effets locaux altiplaniques, particulièrement durant la saison sèche.

L'étude temporelle, entre 1995 et 2004, du signal isotopique montre qu'au cours de la saison des pluies (80 à 90\% de la précipitation s'accumule entre octobre et avril) la variation importante des quantités précipitées est bien marquée dans les teneurs en oxygène $\left({ }^{18} \mathrm{O}\right)$ et par l'excès en deutérium "d" (figure 3). Résultats que l'on retrouve dans l'étude isotopique des pluies de la vallée du Zongo située à une cinquantaine de km de La Paz (Vimeux et al., 2005). Aux précipitations de saison sèche (période mai-septembre) correspondent les teneurs en oxygène 18 les plus élevées, inversement, aux précipitations de saison humide (période octobre-avril) correspondent les teneurs en isotopes lourds les plus faibles.

Sur les 9 cycles annuels considérés, les valeurs isotopiques les plus négatives surviennent, en fin de saison humide (mars-avril) alors que les précipitations mensuelles les plus importantes ont lieu de décembre à février. Le phénomène est lié à l'appauvrissement cumulatif en isotopes lourds des masses d'air océanique, suite aux vidanges successives au dessus du continent sud américain (effet de continentalité). L'effet isotopique au cours de la saison humide apparait donc essentiellement régional. A contrario, les valeurs en isotopes lourds les plus élevées apparaissent en début de saison des pluies (octobre-novembre). Les premières précipitations se produisent au travers de masses d'air de faible teneur en humidité, au moment où les températures sont aussi les plus élevées de l'année, conduisant à des pluies en partie reprises par l'évaporation. Durant cette période, les 
conditions locales jouent un rôle plus important sur la teneur, globalement plus élevée en isotope lourd, des précipitations.

Les événements ENSO impriment aussi leur marque. En conditions El Niño (fort 1997-98 et modéré 2002-04), la dynamique du flux d'Est est alors moins importante et les conditions locales prennent relativement plus d'importance, marquées par des températures plus élevées et des humidités relatives plus basses qu'en situation La Niña. Durant ces épisodes El Niño, on note aussi que l'amplitude de la variation isotopique saisonnière est sensiblement plus faible qu'en situation La Niña $(\approx 12-15 \%$ en oxygène 18 sur la période $1997-98$ et 2004 et jusqu'à $\approx 20 \%$ en oxygène 18 sur la période 2000-01). Les masses d'air sur l'altiplano étant moins saturées durant El Niño, les pluies sont potentiellement enrichies en isotopes lourds $\left({ }^{18} \mathrm{O}, \mathrm{D}\right)$ lors de leur chute à travers l'atmosphère. Inversement en situation La Niña, le flux d'humidité est maximal, l'évaporation durant la pluie est plus limitée et ne masque pas l'héritage du signal isotopique acquis pendant le transfert sur le bassin amazonien en particulier les teneurs les plus négatives. Les valeurs de l'excès en deutérium confirment l'analyse, la variation saisonnière apparaissant nettement plus limitée durant El Niño 97-98 (8\%o) que durant La Niña 2000-01 (14\%o). En général, l'excès en deutérium obéit à la saisonnalité comme l'isotope, les valeurs les plus élevées sont observées au début de la saison humide entre septembre et novembre (notamment durant La Niña 2000-01 où l'on observe des valeurs entre +15 et $+20 \%$ ), les plus faibles au cœur de la saison humide entre janvier et mars, au moment où l'effet local est le plus faible.

Les droites de tendance des teneurs isotopiques ont été calculées de janvier 1996 à décembre 2004 comme pour la température et l'humidité relative (figure 3). La teneur de l'oxygène 18 augmente significativement de 2,6\%, et celle du deutérium (non représentée sur la figure, dans la proportion du lien physique qui les lie) de $19 \%$, celle de l'excès en deutérium diminuant de $0,9 \%$. Aucune conclusion définitive ne peut être avancée, la période de référence n'étant pas assez longue en particulier pour les isotopes. Mais une telle tendance vers un enrichissement en isotopes lourds des 
pluies pourrait correspondre, du moins en partie, à des conditions de réchauffement de l'atmosphère (Kaiser et al., 2002).

La corrélation entre humidité relative et quantité de précipitation à l'échelle mensuelle, $\mathrm{R}^{2}=0,58$ (figure 6 a) est à rapprocher des corrélations oxygène 18 versus précipitation et oxygène 18 versus humidité relative de la figure 4. En général une bonne saison des pluies (1996-97, 1998-99, 200001) est liée à une meilleure saturation de l'atmosphère et inversement une atmosphère moins saturée correspond à une baisse globale de la pluviométrie (1997-98).


Fig. 6 Comparaison hauteur de précipitation mensuelle et a) humidité relative, b) température, à la station de Laïca Cota.

Le dynamisme du flux d'Est conditionne naturellement la qualité de la saison des pluies et l'état de saturation atmosphérique, particulièrement durant les évènements ENSO marqués. En revanche, les températures mensuelles semblent ne présenter aucune corrélation directe avec les précipitations, $\mathrm{R}^{2}=0,10$ (figure $6 \mathrm{~b}$ ), hormis la variabilité intra-saisonnière que l'on retrouve pour les différents paramètres (Vimeux et al 2005). Mais à l'échelle de l'année hydrologique (septembre à août), la température moyenne semble mieux liée aux précipitations, en particulier durant les ENSO, puisqu'une année déficitaire, comme celle du El Niño 1997-98, présente une température moyenne plus élevée $\left(13,5^{\circ} \mathrm{C}\right)$, avec un maximum de $15,5^{\circ} \mathrm{C}$, et qu'inversement une année excédentaire, comme celle de La Niña 1996-97, montre une température moyenne plus faible $\left(11,7^{\circ} \mathrm{C}\right)$, avec un 
maximum de $13,5^{\circ} \mathrm{C}$ (tableau 1). Dans le cas des précipitations saisonnières ce sont aussi les ENSO les plus importants qui produisent les quantités les plus remarquables: dans le sens d'une augmentation pour Les Niña 1996-97 $(631 \mathrm{~mm})$ et 2000-01 $(651 \mathrm{~mm})$ ou d'une baisse pour El Niño 1997-98 (445 mm).

Pour les autres phases ENSO moins marquées (La Niña 1999-01 ou El Niño 2001-02), la relation avec la température est aussi enregistrée, mais dans une moindre mesure, de même que le lien avec la précipitation.

Ces résultats tendent à montrer l'existence d'une relation isotope-climat significative dans la zone d'étude. L'enrichissement isotopique moyen des pluies observé durant la période 1995-2004 pouvant être relié à la fois à la baisse de l'humidité et au réchauffement. En effet, le sens de variation de ces deux paramètres pourrait avoir pour conséquence un enrichissement en isotopes lourds $\left({ }^{18} \mathrm{O} \& \mathrm{D}\right)$ des précipitations locales. Néanmoins, en absence de caractère clairement évaporé des eaux de précipitation sur le diagramme ${ }^{18} \mathrm{O} /{ }^{2} \mathrm{H}$ (figure 5), il est difficile d'attribuer les valeurs plus enrichies et la variation plus grande du rapport isotopique aux seules conditions évaporatoires de l'Altiplano. Car la diminution des précipitations sur le bassin amazonien pourrait très bien, à elle seule, expliquer des teneurs en isotopes lourds plus importantes sans marquage net par l'évaporation, comme c'est le cas.

\section{Hypothèse d'une rémanence de conditions Niño / Niña sur les isotopes des précipitations}

Les moyennes mobiles sur 13 mois ont été calculées sur les données de température, humidité relative et rapports isotopiques (figure 7), afin de gommer la saisonnalité et d'observer, ou non, le synchronisme des paramètres dans les conditions d'une variabilité climatique marquée, celle des évènements ENSO. Cette analyse est particulièrement importante dans la perspective d'utilisation des isotopes comme indicateur de la variabilité du climat, tant pour l'actuel (dans les pluies) que pour le passé (dans les carottes de glace). 


\begin{tabular}{|c|c|c|c|c|c|c|c|c|c|c|c|c|c|c|c|}
\hline An-mois & $\begin{array}{c}\mathrm{P} \\
(\mathrm{mm})\end{array}$ & $\begin{array}{c}\mathrm{T} \\
\left({ }^{\circ} \mathrm{C}\right) \\
\end{array}$ & $\begin{array}{l}\mathrm{Hr} \\
(\%)\end{array}$ & $\begin{array}{c}\delta^{18} \mathrm{O} \\
(\%) \\
\end{array}$ & $\begin{array}{l}\delta^{2} \mathrm{H} \\
(\% 0)\end{array}$ & $\begin{array}{l}\text { "d" } \\
(\%)\end{array}$ & $\begin{array}{l}\mathrm{Bn} \\
(\mathrm{m})\end{array}$ & An-mois & $\begin{array}{c}\mathrm{P} \\
(\mathrm{mm})\end{array}$ & $\begin{array}{c}\mathrm{T} \\
\left({ }^{\circ} \mathrm{C}\right) \\
\end{array}$ & $\begin{array}{c}\mathrm{Hr} \\
(\%) \\
\end{array}$ & $\begin{array}{c}\delta^{18} \mathrm{O} \\
(\%) \\
\end{array}$ & $\begin{array}{l}d^{2} \mathrm{H} \\
(\%)\end{array}$ & $\begin{array}{l}\text { "d" } \\
(\%)\end{array}$ & $\begin{array}{l}\mathrm{Bn} \\
(\mathrm{m})\end{array}$ \\
\hline $1995-9$ & 8,4 & 11,9 & 49 & $-5,60$ & $-33,5$ & 11,30 & $-0,878$ & $2000-5$ & 5,0 & 12,5 & 32 & $-10,17$ & $-74,3$ & 7,06 & \\
\hline 10 & 11,4 & 13,8 & 37 & $-6,41$ & $-39,9$ & 11,38 & & 6 & 21,1 & 10,3 & 28 & $-11,82$ & $-80,2$ & 14,36 & \\
\hline 11 & 38,8 & 13,7 & 40 & $-11,97$ & $-81,2$ & 14,56 & & 7 & 0,2 & 9,8 & 24 & & & & \\
\hline 12 & 114,0 & 12,9 & 49 & $-16,95$ & $-120,1$ & 15,50 & & 8 & 22,1 & 11,5 & 39 & $-9,01$ & $-57,0$ & 15,08 & \\
\hline $1996-1$ & 139,5 & 12,6 & 62 & $-17,05$ & $-122,8$ & 13,60 & & 9 & 1,1 & 13,6 & 31 & $-5,50$ & $-39,3$ & 4,70 & 0,558 \\
\hline 2 & 28,5 & 13,2 & 62 & $-16,52$ & $-122,6$ & 9,56 & & 10 & 62,3 & 13,0 & 45 & $-12,86$ & $-90,7$ & 12,18 & \\
\hline 3 & 38,6 & 13,4 & 54 & $-15,48$ & $-111,7$ & 12,14 & & 11 & 6,3 & 15,1 & 30 & $-4,28$ & $-14,4$ & 19,84 & \\
\hline 4 & 14,3 & 12,8 & 58 & $-21,15$ & $-160,1$ & 9,10 & & 12 & 128,7 & 13,5 & 53 & $-14,68$ & $-108,0$ & 9,44 & \\
\hline 5 & 0,3 & 12,0 & 39 & $-13,55$ & & & & 2001-1 & 179,8 & 11,9 & 75 & $-23,90$ & $-182,1$ & 9,10 & \\
\hline 6 & 1,0 & 10,2 & 29 & $-12,68$ & $-91,9$ & 9,54 & & 2 & 106,9 & 12,6 & 67 & $-17,09$ & $-128,8$ & 7,92 & \\
\hline 7 & 5,4 & 9,7 & 33 & $-9,04$ & $-58,8$ & 13,52 & & 3 & 77,4 & 13,1 & 62 & $-17,45$ & $-129,7$ & 9,85 & \\
\hline 8 & 5,8 & 10,8 & 45 & $-9,25$ & $-64,7$ & 9,30 & & 4 & 20,4 & 13,4 & 42 & $-19,01$ & $-142,4$ & 9,63 & \\
\hline 9 & 22,1 & 11,9 & 43 & $-6,34$ & $-36,0$ & 14,72 & 0,649 & 5 & 20,5 & 12,4 & 32 & $-19,75$ & $-147,9$ & 10,10 & \\
\hline 10 & 19,7 & 13,5 & 44 & $-9,34$ & $-61,2$ & 13,52 & & 6 & 6,5 & 11,2 & 31 & $-9,40$ & $-62,7$ & 12,45 & \\
\hline 11 & 46,6 & 13,0 & 53 & $-13,94$ & $-98,9$ & 12,62 & & 7 & 9,3 & 11,1 & 25 & $-9,41$ & $-59,6$ & 15,63 & \\
\hline 12 & 107,3 & 12,2 & 69 & $-14,11$ & $-101,2$ & 11,68 & & 8 & 31,9 & 10,8 & 33 & $-8,54$ & $-52,1$ & 16,17 & \\
\hline $1997-1$ & 157,9 & 12,7 & 72 & $-12,11$ & $-83,9$ & 12,98 & & 9 & 18,0 & 12,7 & 38 & $-4,50$ & $-19,2$ & 16,75 & $-0,058$ \\
\hline 2 & 76,2 & 11,8 & 74 & $-16,80$ & $-124,7$ & 9,70 & & 10 & 67,3 & 13,3 & 42 & $-8,37$ & $-51,4$ & 15,51 & \\
\hline 3 & 132,2 & 11,3 & 75 & $-17,88$ & $-128,4$ & 14,64 & & 11 & 9,8 & 15,1 & 36 & $-7,00$ & $-44,9$ & 11,10 & \\
\hline 4 & 43,5 & 11,4 & 56 & $-12,48$ & $-87,0$ & 12,84 & & 12 & 75,7 & 13,8 & 43 & $-10,49$ & $-70,4$ & 13,52 & \\
\hline 5 & 17,6 & 10,5 & 44 & $-13,77$ & $-97,7$ & 12,46 & & $2002-1$ & 90,4 & 13,8 & 51 & $-7,99$ & $-45,7$ & 18,22 & \\
\hline 6 & 2,8 & 10,7 & 28 & $-11,99$ & $-86,8$ & 9,12 & & 2 & 117,3 & 13,1 & 54 & $-20,33$ & $-150,7$ & 11,94 & \\
\hline 7 & 0,0 & 11,1 & 32 & & & & & 3 & 80,2 & 13,4 & 56 & $-19,49$ & $-147,1$ & 8,82 & \\
\hline 8 & 5,9 & 10,7 & 46 & $-7,86$ & $-51,7$ & 11,18 & & 4 & 78,9 & 12,7 & 59 & $-18,34$ & $-137,3$ & 9,42 & \\
\hline 9 & 48,1 & 12,4 & 50 & $-10,21$ & $-67,7$ & 13,98 & $-2,173$ & 5 & 12,8 & 12,4 & 41 & $-11,90$ & $-82,5$ & 12,7 & \\
\hline 10 & 37,1 & 14,0 & 41 & $-5,06$ & $-24,9$ & 15,58 & & 6 & 10,6 & 11,2 & 34 & $-11,64$ & $-81,4$ & 11,72 & \\
\hline 11 & 49,6 & 14,2 & 51 & $-11,72$ & $-77,3$ & 16,46 & & 7 & 25,9 & 9,2 & 39 & $-10,89$ & $-73,3$ & 13,82 & \\
\hline 12 & 43,4 & 15,5 & 52 & $-11,92$ & $-85,0$ & 10,31 & & 8 & 10,1 & 11,2 & 29 & $-4,79$ & $-23,1$ & 15,22 & \\
\hline $1998-1$ & 84,3 & 15,1 & 63 & $-10,74$ & $-76,7$ & 9,22 & & 9 & 27,2 & 12,4 & 37 & $-6,72$ & $-40,3$ & 13,46 & $-0,085$ \\
\hline 2 & 62,5 & 14,9 & 69 & $-14,25$ & $-104,4$ & 9,55 & & 10 & 82,6 & 12,3 & 56 & $-6,81$ & $-39,0$ & 15,48 & \\
\hline 3 & 45,1 & 15,0 & 59 & $-19,63$ & $-148,3$ & 8,74 & & 11 & 50,6 & 13,7 & 47 & $-14,09$ & & & \\
\hline 4 & 48,0 & 14,2 & 50 & $-18,35$ & $-137,3$ & 9,50 & & 12 & 63,8 & 13,8 & 47 & $-14,09$ & $-101,8$ & 10,92 & \\
\hline 5 & 0,0 & 12,6 & 24 & & & & & 2003-1 & 134,7 & 13,4 & 62 & $-16,52$ & $-125,5$ & 6,66 & \\
\hline 6 & 19,2 & 10,7 & 36 & $-11,26$ & $-78,7$ & 11,33 & & 2 & 98,9 & 13,5 & 64 & $-11,89$ & $-81,9$ & 13,22 & \\
\hline 7 & 0,0 & 11,0 & 30 & & & & & 3 & 82,8 & 12,9 & 60 & $-20,98$ & $-158,3$ & 9,54 & \\
\hline 8 & 7,4 & 11,9 & 39 & $-5,67$ & $-29,0$ & 16,31 & & 4 & 6,9 & 12,8 & 46 & $-19,95$ & $-153,8$ & 5,80 & \\
\hline 9 & 2,1 & 12,8 & 35 & 1,16 & 22,4 & 13,17 & $-0,450$ & 5 & 5,8 & 11,8 & 46 & $-12,04$ & $-91,0$ & 5,32 & \\
\hline 10 & 57,4 & 13,1 & 50 & $-9,09$ & $-58,1$ & 14,62 & & 6 & 1,6 & 10,9 & 29 & $-6,92$ & $-38,0$ & 17,36 & \\
\hline 11 & 83,3 & 13,4 & 46 & $-14,74$ & $-103,6$ & 14,27 & & 7 & 2,4 & 9,8 & 37 & $-5,24$ & $-27,2$ & 14,72 & \\
\hline 12 & 53,3 & 14,3 & 48 & $-7,17$ & $-40,0$ & 17,31 & & 8 & 8,9 & 10,7 & 39 & $-9,57$ & $-63,2$ & 13,36 & \\
\hline $1999-1$ & 111,1 & 13,0 & 60 & $-12,50$ & $-84,4$ & 15,55 & & 9 & 27,2 & 10,9 & 44 & $-5,79$ & $-25,8$ & 20,52 & $-0,523$ \\
\hline 2 & 48,3 & 12,7 & 70 & $-17,68$ & $-130,5$ & 10,94 & & 10 & 44,3 & 13,3 & 45 & $-9,10$ & $-54,5$ & 18,30 & \\
\hline 3 & 109,4 & 12,2 & 76 & $-17,62$ & $-126,2$ & 14,71 & & 11 & 9,3 & 14,3 & 36 & $-5,24$ & $-28,3$ & 13,62 & \\
\hline 4 & 33,8 & 12,1 & 64 & $-14,06$ & $-99,3$ & 13,13 & & 12 & 109,4 & 14,3 & 53 & $-17,31$ & $-130,5$ & 7,98 & \\
\hline 5 & 5,5 & 11,7 & 38 & $-10,81$ & $-72,9$ & 13,53 & & $2004-1$ & 164,3 & 12,8 & 65 & $-17,25$ & $-126,6$ & 11,40 & \\
\hline 6 & 1,2 & 10,7 & 26 & $-7,71$ & $-47,3$ & 14,33 & & 2 & 61,5 & 13,1 & 58 & $-13,88$ & $-99,8$ & 11,24 & \\
\hline 7 & 7,3 & 10,1 & 35 & $-7,99$ & $-49,2$ & 14,67 & & 3 & 51,0 & 13,7 & 59 & $-17,87$ & $-131,9$ & 11,06 & \\
\hline 8 & 1,0 & 11,1 & 32 & $-2,32$ & $-6,4$ & 12,16 & & 4 & 10,6 & 13,6 & 52 & $-17,64$ & $-129,8$ & 11,32 & \\
\hline 9 & 51,8 & 11,8 & 42 & $-11,70$ & $-75,6$ & 17,95 & 0,076 & 5 & 4,0 & 11,4 & 30 & $-6,70$ & $-45,9$ & 7,77 & \\
\hline 10 & 65,7 & 12,0 & 52 & $-7,57$ & $-44,9$ & 15,61 & & 6 & 6,4 & 10,5 & 34 & $-5,40$ & & & \\
\hline 11 & 26,3 & 13,6 & 38 & $-6,57$ & $-34,0$ & 18,51 & & 7 & 17,7 & 10,0 & 39 & $-7,29$ & $-48,0$ & 10,32 & \\
\hline 12 & 73,4 & 13,8 & 51 & $-12,62$ & $-84,8$ & 16,16 & & 8 & 24,6 & 10,5 & 43 & $-6,28$ & $-36,9$ & 13,34 & \\
\hline $2000-1$ & 109,7 & 12,7 & 62 & $-13,96$ & $-98,1$ & 13,58 & & 9 & 11,5 & 12,1 & 43 & $-1,29$ & $-0,2$ & 10,12 & $-1,693$ \\
\hline 2 & 34,9 & 12,9 & 64 & $-15,47$ & $-110,6$ & 13,11 & & 10 & 15,8 & 14,2 & 54 & $-1,96$ & $-8,1$ & 7,58 & \\
\hline 3 & 72,1 & 13,5 & 59 & $-24,80$ & $-182,3$ & 16,10 & & 11 & 49,5 & 14,5 & 49 & $-8,72$ & $-60,9$ & 8,86 & \\
\hline 4 & 12,6 & 13,4 & 47 & $-9,16$ & $-61,9$ & 11,38 & & 12 & 36,5 & 15,0 & 49 & $-9,53$ & $-66,3$ & 9,94 & \\
\hline Saison & $\begin{array}{c}\mathrm{P} \\
(\mathrm{mm}) \\
\end{array}$ & $\begin{array}{c}\mathrm{T} \\
\left({ }^{\circ} \mathrm{C}\right) \\
\end{array}$ & $\begin{array}{c}\mathrm{Hr} \\
(\%) \\
\end{array}$ & & & & & 95-04 & $\begin{array}{c}\mathrm{P} \\
(\mathrm{mm})\end{array}$ & $\begin{array}{c}\mathrm{T} \\
\left({ }^{\circ} \mathrm{C}\right) \\
\end{array}$ & $\begin{array}{l}\mathrm{Hr} \\
(\%) \\
\end{array}$ & $\begin{array}{c}\delta^{18} \mathrm{O} \\
(\%) \\
\end{array}$ & $\begin{array}{l}\delta^{2} \mathrm{H} \\
(\%) \\
\end{array}$ & $\begin{array}{l}\text { "d" } \\
(\%)\end{array}$ & $\begin{array}{l}\mathrm{Bn} \\
(\mathrm{m})\end{array}$ \\
\hline 95-96 & 406,0 & 12,3 & 46,4 & & & & & Min & 0,0 & 9,2 & 24,0 & $-24,8$ & $-182,3$ & 4,7 & $-2,173$ \\
\hline $96-97$ & 631,8 & 11,7 & 53,0 & & & & & Max & 179,8 & 15,5 & 76,0 & 1,2 & 22,5 & 20,5 & 0,649 \\
\hline $97-98$ & 444,7 & 13,5 & 47,0 & & & & & Moy & 44,2 & 12,5 & 46,8 & $-11,6$ & $-80,7$ & 12,5 & $-0,458$ \\
\hline 98-99 & 513,7 & 12,3 & 48,3 & & & & & ECT & 42,6 & 1,4 & 12,9 & 5,2 & 42,6 & 3,2 & 0,912 \\
\hline 99-00 & 494,9 & 12,3 & 44,8 & & & & & & & & & & & & \\
\hline 00-01 & 651,1 & 12,6 & 43,8 & & & & & & & & & & & & \\
\hline 01-02 & 597,0 & 12,7 & 43,5 & & & & & & & & & & & & \\
\hline 02-03 & 566,2 & 12,3 & 47,5 & & & & & & & & & & & & \\
\hline 03-04 & 530,3 & 12,4 & 46,55 & & & & & & & & & & & & \\
\hline
\end{tabular}

P : Pluie (mm), T : Température $\left({ }^{\circ} \mathrm{C}\right), \mathrm{Hr}:$ Humidité relative $(\%), \delta^{18} \mathrm{O}:$ Oxygène $18\left(\delta \%\right.$ vs SMOW), $\delta^{2} \mathrm{H}$ : Deutérium ( $\delta \%$ vs SMOW), " $\mathrm{d}$ " : Excès en deutérium, ( $\delta \%$ vs SMOW), Bn : Bilan net glacier Zongo (m d'eau) Tableau 1 Données climatiques et isotopiques mensuelles recueillies à Laïca Cota durant la période 1995-2004. Minimum, maximum, moyenne et écart-type sur la période 1995-2004. Moyenne sur l'année hydrologique (septembre à août) pour P, T et Hr. 



Fig. 7 Relation temporelle Pluies mensuelles, Température, Humidité relative, oxygène 18 et excès en deutérium (moyennes mobiles sur 13 mois). L'intensité des S.O.I. mensuels (La Niña quand $<0$ et El Niño si >0) est représentée ainsi que les phases modérées à fortes La Niña et El Niño symbolisées en gris clair et gris foncé respectivement. 
Pour permettre le calcul continu de la moyenne mobile, la série a dû être complétée pour 4 mois de la période (mai 98, juillet 97, 98 et 00) ne présentant pas de valeurs de pluies (tableau 1). Afin de vérifier l'absence de biais dans la correction des données, la valeur mensuelle reconstituée a porté soit sur la moyenne des valeurs brutes de mai et juillet, respectivement, soit sur les valeurs pondérées par la hauteur précipitée. La moyenne mobile résultant de cet aménagement n'apparait pas différente. En ce qui concerne les 6 valeurs d'excès en deutérium manquantes (mai 96, 97, juillet $97,98,00$, novembre 02 , tableau 1), l'ampleur du signal étant plus faible que pour l'oxygène 18, il a été procédé a leur remplacement par la valeur moyenne de l'excès en deutérium sur l'ensemble de la période soit $12,5 \%$.

La figure 7 permet de suivre simultanément l'évolution des signaux isotopiques $\left({ }^{18} \mathrm{O}\right.$ et $\left.\mathrm{d}\right)$, celui des paramètres climatiques (température, humidité, précipitation) et l'indice SOI. Cohérences et décalages coexistent dans l'évolution des signaux :

La période 1996 début 97 qui correspond à un événement La Niña est marquée de façon synchrone par une humidité relative forte (valeurs centrées réduites > 0,5), des températures en baisse (valeurs centrées réduites $<-0,5)$, des teneurs isotopiques des pluies appauvries en isotopes lourds $(\delta 18 \mathrm{O}<$ -13), des excès en deutérium parmi les plus faibles de la série $(\mathrm{d}=+12)$. Cette réaction des paramètres est conforme à une situation de flux d'Est massif qui privilégie le processus de vidange, sans recyclage prononcé depuis l'Amazonie.

Durant El Niño 1997-98, les valeurs de l'humidité relative se maintiennent à un niveau aussi élevé que durant La Niña, pendant la première partie de l'année hydrologique, comme s'il existait un effet de rémanence de cette Niña. L'origine de cette rémanence de l'humidité serait amazonienne et non altiplanique, car ce milieu est nettement trop sec et pauvre en végétation pour induire un tel effet. L'humidité relative diminue ensuite rapidement durant la seconde partie de 1997, les valeurs centrées réduites deviennent négatives, l'effet El Niño s'installe. Cet « effet » de rémanence n'est pas enregistré par les températures, lesquelles sont en effet en constante élévation depuis la fin de l'année 1996 (effet estival de La Niña), et jusqu'à la mi-1998, durant toute la phase El Niño (les 
valeurs centrées réduites passent de $-0,5$ à $+0,7)$. Hormis la variation saisonnière normalement enregistrée la valeur isotopique reste stable au cours de la première partie du El Niño (début 1997 début 1998), autour de $\delta=-12 \%$ pour l'oxygène 18 , avant d'augmenter progressivement jusqu'à début 1999 pour atteindre des valeurs de $\delta=-10 \%$, soit un an après la fin du Niño signalée par les SOI. Comme précédemment pour les paramètres climatiques, il pourrait exister pour les isotopes un effet de rémanence, lié au bassin amazonien. La sécheresse relative installée pendant El Niño sur l'Amazonie maintiendrait un effet sur les teneurs en isotopes lourds des précipitations durant environ un an après la fin des conditions El Niño. L'excès en deutérium, stable pendant le coeur du Niño n'augmente qu'à partir de mi-1998 pour culminer plus tardivement encore que l'oxygène 18 à la fin de la saison sèche de 1999 avec des valeurs approchant $+15 \%$. Il est possible que cet effet plus tardif encore de l'excès en deutérium résulte d'un renforcement des recyclages sur l'Amazonie par une re-évaporation des précipitations hivernales, en ambiance sous-saturée, des contreforts andins à l'Altiplano. Ce n'est donc qu'avec un certain retard, pendant l'année 98 et durant une bonne partie de l'année 1999, que les isotopes vont traduire la mise en place de conditions d'aridité plus sévères correspondant à la phase El Niño: augmentation de l'ordre de deux $\delta$ de l'oxygène-18 des précipitations et plus encore (trois $\delta$ ) de l'excès en deutérium, en accord avec la rémanence affichée des humidités qui semblent préférentiellement gouverner l'isotope.

Durant la seconde phase modérée La Niña mi-1998/2000, la teneur en oxygène 18 des pluies continue à s'appauvrir $\left(\delta^{18} \mathrm{O}=-13,5 \%\right.$ à la fin de l'année 2000) alors que l'excès en deutérium, après être passé par le maximum décrit précédemment, diminue rapidement jusqu'aux valeurs les plus faibles de la période observée $(d=+11 \%$ ). Et cette fois, fin 2000 , l'isotope apparaît bien en phase avec le signal S.O.I.

L'année 2001, est une année de transition, avant une nouvelle phase El Niño faiblement signalée par les S.O.I. L'effet est légèrement marqué à Laïca Cota en termes de température (en hausse) et d'humidité relative (en baisse, hormis la variation saisonnière). Une tendance à l'enrichissement des pluies en isotopes lourds $\left(\delta^{18} \mathrm{O}+2 \%\right.$; $\left.\mathrm{d}+2 \%\right)$ s'installe cependant déjà durant l'année 2001 
signalant bien l'arrivée d'un nouveau «El Niño ». Par la suite, durant les années 2002 à 2004, signalées par les S.O.I. comme étant sous l'influence d'un faible «El Niño », les paramètres climatiques locaux restent stables (hormis la variation saisonnière), alors que le rapport isotopique de l'oxygène des précipitations augmente de $3 \delta$, de $-13 \%$ à $-10 \%$. Cet enrichissement ne peut résulter que de processus antérieurs, et plus lointains, sur la trajectoire des masses d'air.

Il semble en effet que l'explication des décalages ou déphasages, plus d'une fois observés au cours de cette courte période entre les "inputs" ENSO (en particulier celui du El Niño 1997-98), les facteurs climatiques locaux et la réponse du signal isotopique, doive être recherchée dans l'épisode amazonien de transfert des masses d'air atlantiques, avant qu'elles ne pénètrent les Andes. Il est clair que la détermination de la durée d'une phase ENSO dépend du type d'indice utilisé, du type de paramètres définissant l'indice, de la zone de mesure de l'indice. Ainsi, en utilisant les index MEI (Multivariate Enso Index) Niño1-2, Niño3, Niño4, Niño3-4, on constate soit une absence franche de conditions La Niña à la fin de l'année 98, soit une phase tardive ne débutant que vers octobre 99. D'autre part, les indices ENSO rendent compte avant tout des conditions climatiques au-dessus des océans, la réponse sur les continents faisant suite à un déplacement des masses d'air plus ou moins long. C'est le cas en Equateur où malgré une réponse synchrone aux fortes phases El Niño sur la zone côtière (pluviométrie au moins multipliée par 4), la réponse sur les glaciers situés seulement à $250 \mathrm{~km}$ de la côte est retardée d'environ 3 mois (Francou et al., 2004 ; Francou et Vincent, 2007).

Comparativement, ce décalage entre signal isotopique et phase El Niño dans la zone d'étude, tant dans sa mise en place que dans son retrait, pourrait s'expliquer à la fois par la distance qui sépare le signal originel océanique de celui enregistré dans les Andes (entre deux et trois mille km), et par la rugosité engendrée par la végétation amazonienne et les reliefs andins, lesquels favorisent la multiplication des cycles précipitation/évaporation. En ce sens il est certain que l'atmosphère continentale doit présenter une plus grande inertie de réaction et de transfert que l'atmosphère océanique plus réactive aux inputs que constituent les ENSO. D’autre part, si la température dans la zone d'étude apparaît assez synchrone des processus ENSO, ce n'est pas le cas de l'humidité qui 
reste très liée à l'état de la masse d'air d'origine atlantique et à son évolution postérieure amazonienne. A échelle saisonnière les conditions locales apparaissent insuffisantes pour expliquer la variation isotopique. Une recherche de contrôle régional plus large avec modélisation mensuelle des rétro-trajectoires sur l'Amazonie depuis l'Atlantique a montré que les masses d'air subissent une activité convective forte et ralentie au contact des Andes, laquelle serait responsable, en grande partie, de la transformation du signal isotopique océanique (Vimeux et al, 2005).

\section{Impact des conditions ENSO sur l'état du glacier Zongo et les isotopes des pluies}

Le bilan glaciaire net spécifique exprimé sous forme de valeur annuelle (septembre à août) de gain ou perte d'eau du glacier (le bilan mensuel n'est pas calculé sur l'ensemble de la période faute de mesure mensuelle dans la zone d'accumulation du glacier), est présenté en même temps que les valeurs mensuelles de l'isotope et des S.O.I. par la figure 8.

Les bilans glaciaires positifs correspondent aux Niña 1996-97 et 2000-01, ces résultats sont conformes à ceux trouvés dans les Andes Centrales notamment en Equateur (Francou et al., 2004). A ces bilans glaciaires positifs correspondent les valeurs en oxygène 18 des mois de saison des pluies les plus négatives de la période (<-20\%o en avril 96, janvier 2001, mars 2000, février 2002, mars 2003). Un certain synchronisme existe donc entre situation La Niña, bilan glaciaire et comportement isotopique des pluies. Inversement, les bilans glaciaires les plus négatifs correspondent aux périodes El Niño 1997-98 et 2003-04, dans une moindre mesure. Mais ce bilan glaciaire négatif durant un El Niño n'est pas seulement à relier à des précipitations plus faibles qu'en situation La Niña, mais aussi au réchauffement de l'atmosphère qui entraîne sur le glacier un retrait en altitude de la ligne d'équilibre entre zone d'accumulation et d'ablation (ELA). Cette limite fictive correspond plus ou moins à la limite solide/liquide des précipitations, et son déplacement en altitude entraîne une extension de la zone d'ablation où l'albédo est plus faible. La principale conséquence du phénomène pour le glacier est un accroissement de sa fusion. Au bilan glaciaire très négatif du El Niño 1997-98 correspond une nette tendance à l'enrichissement en isotope lourd $\left({ }^{18} \mathrm{O}\right)$ des pluies, qui se poursuit cependant jusqu'au début de l'année 1999, toujours en accord avec 
le bilan net glaciaire négatif du Zongo (Francou et al., 1995 ; 2003), mais en déphasage avec les S.O.I. qui signalent déjà un passage en phase la Niña à la mi-1998.

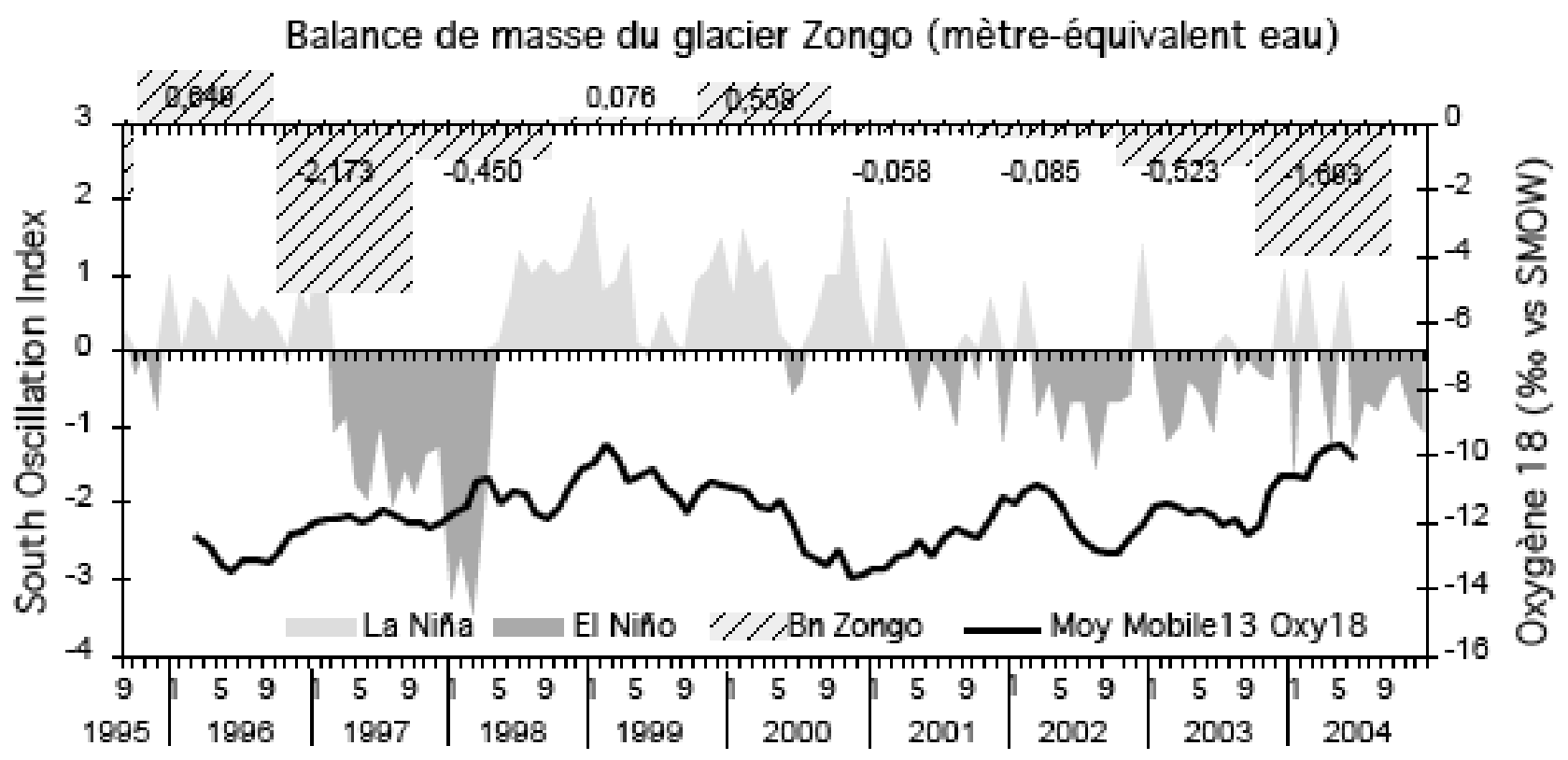

Fig. 8 Relation temporelle S.O.I. mensuels, balance nette annuelle du glacier Zongo et oxygène oxygène 18 (moyennes mobiles sur 13 mois pour ce dernier).

Le bilan glaciaire n'apparaît à nouveau positif qu'en 2000, en accord avec l'oxygène 18 plus négatif, mais alors que le signal S.O.I. de La Niña est déjà plus faible. A partir de mi-2001 les valeurs S.O.I. montrent une nouvelle entrée modérée en phase El Niño, confirmé par un bilan net faiblement négatif du glacier. Ce bilan s'accentue ensuite jusqu'à fin 2004, alors que les teneurs en isotopes lourds des pluies s'enrichissent régulièrement consécutivement à cet effet El Niño continu, à défaut d'être très marqué.

Une étude récente (Vimeux et al, 2005) a montré, utilisant une simulation de rétro-trajectoires des masses d'air, que la variation isotopique interannuelle à la base de la vallée du Zongo, principale entrée des masses d'air amazonien sur l'Altiplano au niveau de La Paz, était contrôlée à 90\% par la distillation résultant des convections répétitives au contact direct de la Cordillère. Par la suite, la composition isotopique des précipitations évolue simplement en fonction de l'altitude et de la baisse des températures $\left(-0,4 \%{ }^{\circ} \mathrm{C}^{-1}\right.$ pour le deutérium) dans cette vallée. C'est sur cette trajectoire N/NO, $\mathrm{S} / \mathrm{SE}$, que les masses d'air plus ou moins ralenties en fonction de l'ENSO, acquerraient l'essentiel 
de l'héritage isotopique enregistré à La Paz. L'augmentation de la teneur isotopique $\left({ }^{18} \mathrm{O}\right)$ des pluies, après une tendance El Niño faible en intensité mais prolongée (trois années), atteint en 2004 une valeur équivalente à celle produite par le fort mais court El Niño 97-98. Le signal isotopique pourrait donc exprimer un effet mémoire et cumulatif susceptible d'expliquer le décalage constaté avec les SOI.

De tels décalages entre conditions climatiques de type ENSO et leurs effets sur les valeurs isotopiques des précipitations doivent être - sur le principe - pris en compte dans les reconstitutions de conditions climatiques à partir d'archives environnementales telles que les carottes de glace. Mais l'incertitude engendrée par ces décalages est de l'ordre de la précision obtenue (un à deux ans), dans la datation des carottes andines relatives au dernier siècle (Vimeux et al, 2005 ; Hoffmann et al, 2003). Cette datation des carottes de glace tropicales découle d'une combinaison de méthodes : sur la partie la plus récente (proche de la surface) de la carotte, la datation est effectuée par comptage des cycles saisonniers secs et humides (sec, plus d'isotopes lourds; humide, moins d'isotopes lourds). Cette datation est contrainte par des événements précis comme les éruptions volcaniques du Pinatubo en 1991, par les pics de tritium ou de césium 137 des expériences nucléaires de 1963, ou par la décroissance radioactive d'éléments comme le plomb 210 (Knüsel et al., 2003). L'erreur étant logiquement croissante avec le temps, la rencontre d'éléments organiques dans la glace qui permettent une datation avec le radiocarbone $\left({ }^{14} \mathrm{C}\right)$ est une aubaine qui permet un recalage. De même la présence de bulles d'air dans la glace est exploitée, car la composition isotopique de l'oxygène est globalement connue suivant les périodes climatiques. Lorsqu'en profondeur (autre extrémité de la carotte) le compactage de la glace ne permet plus de distinguer les cycles saisonniers, la datation est issue de modèles calculant l'évolution de la compaction en fonction de la pression, et par comparaison avec d'autres carottes déjà bien connues.

Les carottes boliviennes seraient ainsi en mesure d'enregistrer grâce aux isotopes les changements de précipitations connectées à l'ENSO, car le rôle des températures semble bien devoir être marginalisé : Connaissant le gradient isotopique, avec la température, de l'élévation des masses 
d'air dans la vallée du Zongo (- 0,4 \%o par ${ }^{\circ} \mathrm{C}$ pour le deutérium), et l'importance de la variation de température moyenne du dernier maximum glaciaire $\left(-2\right.$ à $\left.-4{ }^{\circ} \mathrm{C}\right)$, seulement $11 \%$ de la variation isotopique enregistrée par les carottes pourraient être expliqués par la variation de température liée à l'altitude. Le principe de «thermo-dépendance» des isotopes des précipitations doit donc bien être relativisé dans les Andes.

\section{CONCLUSION}

Si les isotopes des pluies sont bien le reflet de l'histoire d'une masse d'air de son origine à son lieu de précipitation, il ne semble pas que les conditions locales d'altitude et d'aridité de l'Altiplano bolivien aient eu la possibilité de modifier la signature isotopique des précipitations de La Paz. La teneur isotopique de ces précipitations serait l'héritage direct des derniers transferts convectifs au contact des Andes péruviennes et boliviennes.

Malgré l'alternance constatée à Laïca Cota, au cours des dernières années, des phénomènes NiñoNiña, signalés par les S.O.I., comme par les teneurs isotopiques des précipitations, la tendance climatique au réchauffement et à l'assèchement des masses d'air se poursuit sur l'altiplano bolivien. Ce fait est confirmé par la fusion glaciaire, qui est une réalité de terrain, comme par la modélisation des températures. Les glaciers tropicaux andins se comporteraient ainsi comme de véritables "thermomètres climatiques" régionaux, et la variation de la teneur isotopique des pluies est bien concomitante de celle du bilan de masse du glacier Zongo.

Un certain synchronisme est observable entre enrichissement / appauvrissement en isotopes lourds et phase El Niño 1997-98 et 2002-04 / La Niña, 1996-97 et 1998-01. Cependant il semble que la concordance de temps soit meilleure durant les Niña (1996-97, et dans une moindre mesure 199901). Le signal S.O.I., qui est un signal océanique du Pacifique, pourrait signaler plus promptement l'arrivée d'un phénomène La Niña, dans la mesure où c'est l'affaiblissement de la circulation d'ouest pacifique qui semble stimuler l'entrée d'air atlantique sur le bassin amazonien (ou vice versa). L'installation de conditions El Niño fortes semble en revanche perturber l'atmosphère 
continentale sur l'altiplano bolivien jusque plus d'un an après que les indices ENSO aient signalé sa fin sous l'effet d'une rémanence des conditions antérieures sur le bassin amazonien. L'excès en deutérium réagit globalement de manière identique, augmentation / diminution en phase El Niño / La Niña, moins en relation avec les conditions atmosphériques locales qu'en raison de l'héritage amazonien. Les paramètres climatiques locaux, $\mathrm{T}$ et $\mathrm{Hr}$, varient de façon synchrone et prononcée lors des forts événements ENSO (La Niña 1996-97 et El Niño 1997-98), de manière beaucoup plus faible lors des événements ENSO moins marqués.

Ces résultats, montrent aussi que le décalage de quelques mois à un an enregistré entre les signaux isotopiques et l'ENSO n'altère en rien l'interprétation de l'archive climatique constituée à partir de l'analyse des isotopes de l'eau des carottes de glace. Car ce décalage est généralement couvert par les incertitudes diverses liées au découpage de la carotte, à l'analyse, à l'interprétation, qui ne permettent de dater le dernier siècle qu'à \pm 2 ans dans les Andes. Cette précision est néanmoins, sous les tropiques, beaucoup plus grande qu'aux hautes latitudes où elle n'est que de l'ordre du siècle.

Remerciements: Les auteurs remercient l'Observatoire géophysique de San Calixto (La Paz), pour la disponibilité de ses données météorologiques ainsi que Françoise Vimeux du LSCE (IRD/HSM Saclay), Patrick Ginot et Christophe Stürm (IRD/LGGE Grenoble) pour leurs conseils qui ont permis d'améliorer ce travail.

\section{REFERENCES}

Craig, H. (1961) Isotopic variations in meteoric waters. Sciences 133, 1702-1703.

Dansgaard, W. (1964) Stable isotopes in precipitation. Tellus 16, 435-468.

Espinoza, J.C., Ronchail, J., Guyot, J.L., Filizola, N., Noriega, L., Ordonez Galves J.J., Pombosa, R. \& Romero, H. (2009) Spatio - Temporal rainfall variability in the Amazon Basin 
Countries (Brazil, Peru, Bolivia, Colombia and Ecuador). International Journal of Climatology, 29, 11, 1574-1594..

Francou, B., Ribstein, P., Sémiond, H., Portocarrero, C. \& Rodriguez, A. (1995) Balances de glaciares y clima en Bolivia y Perú: impacto de los eventos ENSO. Bull. Inst. Fr. Etudes Andines 24 (3), 661-670.

Francou, B., Vuille, M., Wagnon, P., Sicart, J. E. \& Mendoza, J. (2003) Tropical climate change recorded by a glacier during the last decades of the XXth century: Chacaltaya, Bolivia, $16^{\circ} \mathrm{S}$. J. Geophys. Res. 108, D5, 1.1-1.12.

Francou, B., Vuille, M. Favier, V., Cáceres, B. (2004) New evidence for an ENSO impact on low-latitude glaciers: Antizana 15, Andes of Ecuador, 0²8'S. J. Geophys. Res. 109(D18) doi :10.1029/2003JD004484.

Francou, B., Pouyaud, B. \& col. (2004) Métodos de observación de glaciares en los Andes Tropicales : Mediciones de terreno y procesamiento de datos, éd. IRD, 243p.

Francou, B. \& Vincent, C. (2007) Les glaciers à l'épreuve du climat. IRD édition et Belin édition, 274 .

Garreaud, R., Vuille, M. \& Clement, A.C. (2003) The climate of the Altiplano: Observed current conditions and mechanisms of past changes. Palaeo. 194, 5-22.

Gat, J.R. \& Matsui, E. (1991) Atmospheric water balance in the Amazon Basin: An Isotopic evapotranspiration model. J. Geophys. Res. 96, 13, 179-13,188.

Gonfiantini, R., Roche M.A., Olivry J.C, Fontes J.Ch. \& Zuppi G.M. (2001) The altitude effect on the isotopic composition of tropical rains. Chem. Geol., 181, 147-167.

Hoffmann, G., Ramirez, E., Taupin, J.D., Francou, B., Ribstein, P., Delmas, R., Dürr, H., Gallaire, R., Simões, J., Schotterer, U., Stievenard, M. \& Werner, M. (2003) Coherent isotope history of Andean ice cores over the last century. Geophys. Res. Lett. 30 (4), 1179-1182.

IPCC (2007) Summary for policymakers. IPCC WGI Fourth Assessment Report, 18p.

Kaiser, A., Scheifinger, H., Kralik, M., Papesch, W., Rank, D. \& Stichler W. (2002) Links between meteorological conditions and spatial/temporal variations in long-term isotope records from 
the Austrian precipitation network. In: Study of environmental change using isotope techniques, 23-27 April 2001, Vienna, ed. by IAEA, C\&S papers Series 13/P, 67-76.

Kalnay, E., Kanamitsu M., Kistler R., Collins W., Deaven D., Gandin L., Iredell M., Saha S., White G., Woolen J., Zhu Y., Chelliah M., Ebisuzaki W., Higgins W., Janowiak J., Mo K. C., Ropelewski C., Wang J., Leetmaa A., Reynolds R., Jenne R \& Joseph D. (1996) The NCEP/NCAR 40-years reanalysis project. Bull. Am. Meteor. Soc., 77, 437-472.

Knüsel, S., Ginot, P., Schotterer, U., Schwikowski, M., Gäggeler, H.W., Francou, B., Petit, J.R., Simoes, J.C. \& Taupin, J.D. (2003) Dating of two nearby cores from the Illimani, Bolivie. $J$. Geophys. Res. 108, D6, 3.1-3.10.

Merlivat, L. \& Jouzel, J. (1979) Global climatic interpretation of the deuterium-oxygen18 relationship for precipitation. J. Geophys. Res. 84, D7, 5029-5033.

Pouyaud, B., Ribstein, P., Gallaire, R., Chevallier, P., Caballero, Y., Berthier E. (1999) Régimes hydrologiques des hauts bassins nivo-glaciaires boliviens et variabilité pluviométrique des vallées andines en conditions El Niño et La Niña. In: Manaus'99, Hydrological and Geochemical processes in large scale River basins. Ed. J.L. Guyot, Manaus 16-19 novembre 1999, Cd rom.

Pouyaud, B., Zapata, M., Yerren, J., Gomez, J., Rosas, G., Suarez, W. \& Ribstein, P. (2005) Avenir des ressources en eau glaciaire de la Cordillère Blanche. Hydrol. Sci. J. 50, 999-1022.

Ribstein, P., Rigaudière, P., Francou, B. \& Saravia, R. (1996) Variabilidad climática y modelación hidrológica del Glaciar Zongo, Bolivia. Aguas, Glaciares y cambios climáticos en los Andes Tropicales. Seminario Internacional, 13-16 de junio 1995, La Paz, Bolivia (ed. by B.Francou \& P. Ribstein) In: Bull. Inst. Fr. Etudes Andines 24, 3,639-649.

Ronchail, J. \& Gallaire, R. (2006) ENSO and rainfall along the Zongo valley (Bolivia) from the Altiplano to the Amazon basin, Int. J. Climatol. 26, 1223-1236.

Rosanski, K. \& Araguas-Araguas, L. (1995) Spatial and temporal variability of stable isotope composition of precipitation over the South American continent. Seminario Internacional, 13- 
16 de junio 1995, La Paz, Bolivia (ed. by B.Francou \& P. Ribstein) In: Bull. Inst. Fr. Etudes Andines 24, 3, 379-390.

Salati, E., Dall'Olio, A., Matsui, E. \& Gat, J.R. (1979) Recycling of water in the Amazon Basin: An isotopic study. Water Resour. Res. 15, 1250-1258.

Segaline Nieto, H., Cabre, R. (1988) El clima de La Paz, datos del observatorio San Calixto, bodas de diamante 1913-1988. La Paz Bolivia Ed. s.n., 80p.

Sicart, J. E., Ribstein, P., Francou, B. \& Gallaire, R. (2003) Etude des précipitations et de la fonte sur un glacier tropical : Le glacier du Zongo, Bolivie Hydrol. Sci. J. 48(5), 799-808.

Sicart, J. E., Ribstein, P., Francou, B., Pouyaud B. \& Condom T. (2007) Hydrological and glaciological mass balances of a tropical glacier: Zongo, Bolivia. Glob. Planet. 59(1), 27-36..

Taupin, J.D., Coudrain-Ribstein, A., Gallaire, R., Zuppi, G.M. \& Filly, A. (2000) Rainfall characteristics $\left(\delta^{18} \mathrm{O}, \delta^{2} \mathrm{H}, \Delta \mathrm{T}\right.$ and $\left.\Delta \mathrm{H}_{\mathrm{r}}\right)$ in Western Africa, regional scale and influence of irrigated areas.. J. Geophys. Res. 105, 11911-11924.

Vimeux, F., Gallaire, R., Bony, S., Hoffmann, G. \& Chiang, J.C.H. (2005) What are the climate controls on $\delta \mathrm{D}$ in precipitation in the Zongo valley (Bolivia)? Implications for the Illimani ice core interpretation. Earth Planet. Sci. Lett. 240, 205-220.

Vuille, M., Bradley R. \& Keimig, F. (2000) Interannual climate variability in the Central Andes and its relation to tropical Pacific forcing. J. Geophys. Res, 105, 12447-12460.

Wagnon, P., Ribstein, P., Francou, B., Sicart, J.E., (2001) Anomalous heat and mass budget of glacier Zongo, Bolivia, during the 1997/1998 El Niño year. J. of Glaciology, 47, nº156.

World Meteorological Organization (1956) International Cloud Atlas, Vol.1, ed. By W.M.O.,155p. 